\title{
L'évolution de l'enseignement des sciences physiques et chimiques dans l'enseignement secondaire agricole : un regard institutionnel
}

\author{
N.HERVE \\ C. DUCAMP
}

\section{Introduction}

Depuis la loi sur la modernisation agricole de 1969 jusqu'à la loi d'avenir pour l'agriculture de 2014, les mondes agricole, scientifique et scolaire ont changé et changent encore.

L'objet de ce chapitre est de spécifier ce changement et de rendre compte de l'évolution de la discipline scolaire des sciences physiques et chimiques dans l'enseignement secondaire agricole. Cette discipline relève à la fois d'un enseignement général, dont l'objectif se décline souvent en «culture scientifique et technologique» pour tous, et d'un enseignement technique agricole dont elle constitue une partie des références théoriques (par exemple la physique pour le machinisme agricole et la chimie pour l'agronomie). Nous interrogeons donc ici cette double dimension, en prenant une approche historique. En effet, selon la formule de Durkheim (1922, p. 49), « pour pouvoir anticiper ce que le présent doit devenir, tout comme pour pouvoir le comprendre, il nous faut en sortir et nous retourner vers le passé ». C'est donc avec l'idée de mieux comprendre les enjeux du présent que nous nous tournons vers le passé, car «pour pouvoir remplir, comme il convient, notre fonction dans un système scolaire, quel qu'il soit, [qu'] il faut le connaître, non du dehors, mais du dedans, c'est-à-dire par l'histoire. Car, seule, l'histoire peut pénétrer au-delà du revêtement superficiel qui le recouvre dans le présent ; seule, elle en peut faire l'analyse ». Nous présentons tout d'abord quelques éléments de contexte qui justifient notre questionnement et développons les concepts permettant de caractériser l'évolution de la discipline. Nous précisons ensuite comment nous avons mis en relation un ensemble de témoignages et de documents d'archives, et rendons compte de l'analyse que nous en avons faite.

\section{Du plan de modernisation agricole à «produire autrement »: un changement de regards sur les sciences et techniques}

\subsection{La place centrale des savoirs scientifiques et techniques dans la modernisation de l'Agriculture}

Si la modernisation de l'agriculture est un impératif politique et économique au début des années 1960, c'est bien par rapport aux sciences et techniques qu'on jauge cette modernisation, notamment par comparaison avec l'industrie qui s'est dotée après la deuxième guerre mondiale de techniques pointues : c'est ainsi que Klatzmann (1953, p. 652) faisait remarquer que «l'agriculture constitue le secteur de la production le plus en retard en ce qui concerne le niveau technique. C'est dans l'agriculture qu'il y a le plus de progrès à réaliser, que la productivité peut le plus augmenter ». Dans le même article, il donne à voir l'image idéale d'une agriculture « moderne » : « on peut rêver une agriculture prospère dont le visage pourrait être le suivant: des agriculteurs instruits disposant de sols améliorés - amendés, drainés, irrigués, remembrés- bien équipés en matériel, utilisant en abondance engrais et semences sélectionnées, vivant dans des exploitations aux bâtiments modernes et bien aménagés » (Klatzmann, 1953, p. 646). Les sciences et techniques sont ainsi centrales dans les discours mettant en place la modernisation du monde agricole : ce sont les méthodes des 
sciences physiques et chimiques qui fondent les approches techniques agronomiques (appelée aussi phytotechniques) et de machinisme agricole, et ce sont donc ces sciences qui doivent être au cœur de l'effort de formation technique, « selon les orientations données par la loi de 1882 la formation doit être «fondée sur les sciences physiques et naturelles appliquées à l'agriculture ", expression que l'on retrouve dans tous les manuels de 1898 à 1960 » (Bonneviale et Sebillote, 1985, p. 36). La loi du 2 Août 1960 sur la formation professionnelle agricole va concrétiser ce projet, et, dans le même temps, il va constituer le terreau d'une approche scientifique des techniques de production, qui deviendra indépendant du modèle que constituent les sciences physiques et chimiques : «depuis que l'agriculture est considérée comme l'application des sciences physiques et naturelles il y a eu réticence à théoriser en agronomie. Ce n'est que depuis les années 60 que le nouveau courant agronomique tend à renverser cette tendance » (Bonneviale et Sebillote, 1985, p. 41). Nous précisons dans les paragraphes qui suivent l'évolution des liens entre sciences et techniques au cours du 20 ème siècle.

\subsection{Une évolution du rapport entre les sciences et les techniques dans le domaine agricole}

Le début du $20^{\text {ème }}$ siècle hérite de la hiérarchie des savoirs issue du positivisme, qui postule que l'ordre des connaissances est dominé par les connaissances scientifiques théoriques et que les savoirs «pratiques » sont seconds et dérivés de la théorie. Pour Fourez (1994), cette division historique entre les sciences «pures» ou «académiques » issues des savoirs universitaires, et les sciences «appliquées » (celles des écoles professionnalisantes de la fin $\mathrm{du} 19^{\text {ème }}$ siècle) provient de la valorisation accordée à un travail qui s'éloigne du travail manuel. Cette perspective irrigue les savoirs scientifiques et techniques scolaires. L'analyse que Bonneviale et Sebillote (Bonneviale et Sebillote, 1985, p. 37) font des livres d'agronomie de 1890 à 1960 montre en effet que ces ouvrages visent à « inculquer des normes à partir d'un discours scientifique qui leur sert de «base» et plus encore de justificatif pédagogique et idéologique, en effet l'agriculture a beau être le plus beau métier il est «naturellement» dépendant... des sciences physiques et naturelles ».

Toutefois, la reconnaissance sociale accordée aux sciences physiques et chimiques sur les techniques agricoles va être remise en cause progressivement. D'un point de vue épistémologique d'abord, il apparaît que «la connaissance scientifique n'est pas le système conceptuel susceptible d'assurer en lui-même la maîtrise technique » (Darré, 1985). Ainsi, les pratiques ne dérivent pas uniquement des savoirs théoriques et ce mouvement s'inscrit dans la reconnaissance d'une complexification des liens entre sciences et techniques : «actuellement la tendance est de considérer que la formation doit faire acquérir les méthodes scientifiques permettant de guider l'action. Pour cela il est nécessaire que l'élève acquière des méthodes d'investigation non seulement relatives à des liens de causes à effets, mais plus encore relatives à des relations faisant appel à des rétroactions, à des interactions entre facteurs de façon à pouvoir développer une analyse en terme de système face aux phénomènes complexes qui se déroulent tant dans la parcelle cultivée que dans l'exploitation agricole ou même dans la cité » (Bonneviale et Sebillote, 1985, p. 42).

D'un point de vue de l'efficacité professionnelle ensuite, Dascon (1985) montre que les savoirs à enseigner relèvent davantage de « connaissances élaborées par la « technostructure » qu'à la réalité quotidienne des jeunes agriculteurs ». Autrement dit, les savoirs scientifiques ne trouvent pas leur légitimité vis-à-vis des milieux professionnels dans lesquels ils sont sensés s'implanter.

A l'heure actuelle, les pratiques fondées sur les connaissances exclusivement scientifiques sont questionnées et les savoirs «tacites » ou bien «sauvages » (Darré, 1996) sont valorisés pour accéder à de « bonnes pratiques agricoles ». 


\subsection{Produire autrement : un nouveau rapport entre sciences et techniques}

La question de la modernisation de l'agriculture française a évolué depuis 1960. Elle n'a plus pour objectif unique l'augmentation de la production agricole : «la nécessité de conserver une base productive capable de participer aux équilibres alimentaires mondiaux a maintenant rejoint, au rang des priorités politiques, celles d'assurer un haut niveau de sécurité sanitaire pour les consommateurs et de réduire les impacts négatifs de l'agriculture sur les milieux naturels. L'imbrication de ces trois objectifs implique la conduite de l'action publique dans un cadre intégré qui soit en capacité d'agir « de la fourche à la fourchette ». De 1960 à 1990, les politiques publiques en faveur de l'agriculture ont donné la priorité au développement économique dans un cadre national et dans une Europe en construction. De 1990 à nos jours, ces priorités ont été infléchies dans le sens d'un développement productif plus durable, dans le cadre d'une mondialisation accélérée des échanges » (Assemblée Nationale, 2013, p. 3 ).

Il s'agit donc de moderniser l'agriculture en passant d'une agriculture majoritairement intensive à une agriculture plus durable, ce qui nécessite « une modification majeure des cadres de pensée et des modes d'acquisition des savoirs des producteurs et de leur accompagnement. Ces changements portent sur un pas de temps long et l'enseignement agricole doit être en mesure de prendre en compte de façon très précoce les évolutions de connaissances, de modes de raisonnement et de pratiques »(DGER, 2013, p. 1). Les sciences et les techniques voient ainsi leurs frontières traditionnelles bouleversées : "l'enseignement supérieur agricole est au cœur du rapprochement entre biologie, écologie et santé, les sciences et technologies du vivant et de l'environnement dépassent les frontières des disciplines historiquement tournées vers la production (agronomie, sciences forestières, zootechnie) pour proposer ingénierie environnementale et agroécologique, procédés et produits, recherches cliniques, ainsi que des dispositifs épidémiologiques ou d'évaluation des risques répondant aux défis sociétaux, en recourant, le cas échéant, à des disciplines plus éloignées, mathématiques et sciences de l'Homme pour tenir compte de l'incertitude de ces systèmes complexes » (Assemblée Nationale, 2013, p. 29). Ce sont donc des savoirs pluriels qui sont convoqués, dont l'articulation n'est pas stabilisée, et qui répondent à des problèmes complexes en lien avec la gestion de l'incertitude.

Dans ce contexte, nous nous interrogeons sur la transposition didactique, au sens de Chevallard (1991), des savoirs scientifiques et techniques, et notamment la forme disciplinaire qu'elle peut prendre actuellement dans l'enseignement technique agricole.

\subsection{Quelle évolution de la discipline «sciences physiques et chimiques» dans un enseignement technique agricole?}

Si les sciences et les techniques ont été mises au centre de la modernisation agricole de 1960, et que le rapport entre les sciences et les techniques a évolué, il nous semble intéressant d'étudier la trajectoire des savoirs scientifiques et techniques scolaires : comment ces savoirs vivent-ils ou se stabilisent-ils dans des programmes scolaires, alors que l'écosystème dans lequel ils vivent se modifie?

Pour aborder cette problématique, nous nous centrerons sur l'évolution des sciences physiques et chimiques, en tant que discipline scolaire de l'enseignement secondaire agricole. Quelle place a eu et a actuellement l'enseignement des sciences physiques et chimiques dans l'enseignement secondaire agricole ? Les savoirs à enseigner et les méthodes pédagogiques des sciences physiques et chimiques sont-ils stables ou ont-ils changé ? Aussi, nous aborderons ces questions sous l'angle des prescriptions, qui donnent à voir les orientations données par l'institution. 
Il nous semble d'autant plus nécessaire de prendre ce regard historique qu'il se construit actuellement un nouveau contrat social sur les pratiques agricoles, dans lequel de nouvelles références scientifiques et techniques sont mises en avant (l'agro-écologie par exemple). Comment la discipline scolaire des sciences physiques et chimiques peut-elle contribuer à alimenter ce changement, et le peut-elle ?

Afin de répondre à ces questions, nous précisons quelques éléments théoriques qui vont nous permettre de décrire l'évolution de la discipline scolaire des sciences physiques et chimiques.

\section{Caractérisation d'une discipline scolaire et description de son évolution}

Nous développons dans cette partie le concept d'épistémologie scolaire que nous mobilisons par la suite afin de décrire la discipline scolaire des sciences physiques et chimiques. Nous référons également notre démarche aux méthodes de l'histoire du temps présent, afin de nous donner les moyens de suivre l'évolution récente de cette discipline.

\subsection{L'épistémologie scolaire : un concept pour décrire une discipline scolaire}

Develay (1993) conçoit la notion d'épistémologie scolaire comme un outil d'analyse permettant de rendre compte des caractéristiques d'une discipline scolaire : «le propos d'une épistémologie scolaire est ainsi de proposer un modèle commun pour analyser la diversité des contenus d'enseignement. Si l'on souhaite une concertation entre enseignants de différentes disciplines, il devient nécessaire de proposer des outils qui leur soient communs, au-delà des différences entre les contenus qu'ils ont à enseigner» (Develay, 1993, p. 37). Pour Roure (2013, p. 167), «il s'agit de porter un regard critique, réflexif et distancié sur les savoirs scolaires d'une discipline (...) pour caractériser et comprendre leurs évolutions, afin d'envisager leurs sens pour les élèves et l'institution scolaire ».

Develay (1993) caractérise ainsi une discipline scolaire par 5 dimensions :

- Les objets manipulés renvoient à tous les objets matériels qui sont manipulés par l'enseignant et/ou les élèves dans l'enseignement d'une discipline scolaire particulière. Develay (1993, p. 38) lie l'évolution dans le temps de l'utilisation des objets avec l'évolution des contenus de savoir enseignés : « une discipline évolue en même temps que les objets qu'elle manipule ».

- Les tâches prescrites dans une discipline relèvent de ce que l'activité des élèves y a de spécifique : c'est-à-dire que font les élèves et dans quel but. C'est une notion qui selon Develay relève des spécificités d'une discipline et constitue également un marqueur temporel de l'évolution de la discipline.

- Les connaissances déclaratives sont les savoirs à enseigner qu'une discipline se charge d'institutionnaliser. Develay (1993) distingue plusieurs classes de connaissances déclaratives : des faits, qui sont des énoncés spécifiques qu'il s'agit de mémoriser (par exemple les dates en histoire, les lois en science, etc.) ; des notions qui donnent sens aux faits (par exemple la notion de révolution en histoire, la notion de gaz en physique, etc.); des registres de conceptualisation qui marquent le niveau conceptuel d'une notion dans une classe donnée (on n'aborde pas les gaz avec le même niveau conceptuel à l'école primaire et au lycée); des champs notionnels qui sont les liens que les notions entretiennent entre elles (par exemple, la notion de gaz et la notion d'atomes, la notion de gaz et la notion de température) ; enfin Develay (1993) considère que quelques concepts intégrateurs sont «les clés de lecture » d'une discipline donnée, à un niveau donné. 
- Les connaissances procédurales sont «de l'ordre des méthodes, techniques, stratégies » utilisées par les élèves et l'enseignant dans une discipline donnée (Develay, 1993, p. 39).

- La matrice disciplinaire est la résultante des 4 dimensions précédentes et correspond au principe organisateur de la discipline : «une matrice disciplinaire représente un modèle de pensée, une vision de la discipline, partagée par les acteurs de l'enseignement » (Roure, 2013, p. 170).

L'objectif de ce chapitre est donc de décrire l'évolution de l'épistémologie scolaire de la discipline «sciences physiques et chimiques » de l'enseignement agricole, afin de mieux comprendre la dialectique sciences / techniques dans un enseignement de type professionnel à partir du cas particulier de cette discipline. Toutefois, cette mise en perspective de type historique se fait dans le contexte actuel rempli d'incertitudes. C'est ce contexte qui nourrit ce projet et il nous semble nécessaire de clarifier l'objectif que nous poursuivons pour éclairer l'action présente.

\subsection{Décrire l'évolution de l'épistémologie scolaire : une histoire du temps présent}

En tant que formateurs d'enseignants de Physique-Chimie de l'Enseignement Agricole, nous sommes sensibles aux changements à la fois dans le métier d'enseignant (multiples réformes ces dernières années, $\mathrm{RVP}^{1}, \mathrm{BTSA}^{2}, \mathrm{AP}^{3}$, livret de compétences en $3^{\mathrm{e}}$, évaluation par capacités en baccalauréat professionnel et BTSA), dans le métier d'enseignant des sciences physiques et chimiques (nouveaux horaires, nouvelle répartition modulaire des référentiels de formation, nouvelles démarches pédagogiques, nouveaux outils) et dans la professionnalisation des enseignants (nouveau plan de formation en lien avec l'émergence des ESPE $^{4}$ ). Ces multiples changements créent de l'incertitude et nous poussent à regarder le passé, non seulement pour prendre en compte la continuité qui lie les événements du passé aux événements présents, mais aussi pour donner de l'épaisseur temporelle aux changements présents, afin de permettre au présent d'être «peu à peu délivré de son autisme » (Rioux, 1992, p. 54).

Cette ambition s'apparente à un travail d'histoire. Mais, nous ne sommes pas historiens, aussi prenons-nous quelques précautions, qui sont autant de points de vigilance pour nous comme pour le lecteur.

Tout d'abord, s'intéresser à l'évolution de l'enseignement des sciences physiques et chimiques dans l'enseignement agricole, c'est s'intéresser à une période historique récente, ce qui fait que ce travail s'inscrit dans une démarche de type " histoire du temps présent », que Wolikow (1998, p. 14) définit comme «une séquence historique définie en amont par la première génération des survivants et en aval par le monde actuel ». La particularité de cette approche tient à l'utilisation du témoignage en tant que source historique produite par le chercheur : "l'histoire du temps présent est donc d'abord celle qui se fait avec et sous le contrôle de témoins, c'est également celle qui peut utiliser leur contribution pour pallier l'absence de certaines archives scripturales et pour en constituer d'autres qui permettent d'écrire l'histoire autrement » (Wolikow, 1998, p. 14).

Travailler sur l'histoire du temps présent, c'est donc à la fois sélectionner des traces historiques issues d'archives mais également produire des traces à partir de témoignages.

Ce recueil d'expériences humaines, sous forme d'entretiens par exemple, permet un dialogue dans le temps entre des acteurs qui n'ont pas vécu la même chose, dont les projections dans le

\footnotetext{
${ }^{1}$ Rénovation de la Voie Professionnelle

${ }^{2}$ Brevet de Technicien Supérieur Agricole

${ }^{3}$ Accompagnement Personnalisé

${ }^{4}$ Ecole Supérieure du Professorat et de l'Enseignement
} 
passé et le futur sont différentes, mais qui ont en commun d'avoir participé ou de participer encore à la vie d'une même institution, dans notre cas l'institution liée à l'enseignement des sciences physiques et chimiques dans l'enseignement agricole. Ce dialogue entre générations a pour finalité de prendre en compte le temps passé afin de donner des pistes d'action pour le présent.

Il s'agit donc pour nous de coucher à l'écrit un récit et nous reprenons à notre compte le projet de Ricœur (1983, p. 435) : «notre hypothèse de travail revient ainsi à tenir le récit pour le gardien du temps, dans la mesure où il ne serait de temps pensé que raconté ». Ce récit à construire constitue donc, en suivant Ricœur, une mise en intrigue de divers éléments afin d'interpréter de notre point de vue comment le passé éclaire le présent.

\section{L'articulation de témoignages et de documents d'archives}

Le cœur de notre méthode (entretiens semi directifs, analyse de documents, analyse de discours, analyse de curriculums) consiste dans l'articulation de traces produites, ici des témoignages, et des documents d'archives afin d'étudier l'évolution des prescriptions de l'enseignement des sciences physiques et chimiques dans l'enseignement secondaire agricole. Nous avons tout d'abord organisé des entretiens semi-directifs de 6 inspecteurs des sciences physiques et chimiques, en activité ou à la retraite, que nous avons sollicités pour témoigner de l'évolution de l'enseignement de cette discipline. Toutes ces personnes ont été également enseignantes et certaines d'entre elles ont formé des enseignants.

Nous décrivons dans le tableau $\mathrm{n}^{\circ} 1$ la temporalité des carrières des personnes interrogées.

\begin{tabular}{|c|c|c|c|c|c|c|}
\hline $\begin{array}{c}\text { Identification } \\
\text { de la personne }\end{array}$ & $\mathrm{I}_{1}$ & $\mathrm{I}_{2}$ & $\mathrm{I}_{3}$ & $\mathrm{I}_{4}$ & $\mathrm{I}_{5}$ & $\mathrm{I}_{6}$ \\
\hline $\begin{array}{c}\text { Date d'entrée } \\
\text { dans l'EA }\end{array}$ & 1969 & 1970 & 1970 & 1980 & 1979 & 2008 \\
\hline $\begin{array}{c}\text { Date de sortie } \\
\text { de l'EA }\end{array}$ & 2006 & 2006 & 2007 & $\begin{array}{c}\text { En cours } \\
\text { d'activité }\end{array}$ & $\begin{array}{c}\text { En cours } \\
\text { d'activité }\end{array}$ & $\begin{array}{c}\text { En cours } \\
\text { d'activité }\end{array}$ \\
\hline
\end{tabular}

Tableau n ${ }^{\circ} 1$

L'entretien visait à documenter les différentes dimensions de l'épistémologie scolaire définie par Develay (1993) et portait ainsi sur les points suivants :

- La carrière: il s'agissait de situer l'expérience humaine dans sa temporalité, l'évolution de la personne dans l'institution ;

- La description de l'enseignement agricole à l'époque de leur entrée dans cette institution, ainsi que la place des sciences physiques et chimiques : ces éléments contribuaient à remonter le temps de la mémoire et à situer le ressenti des personnes interrogées ;

- L'évolution de l'architecture des diplômes, les points-clés d'évolution de l'enseignement des sciences physiques et chimiques au cours de leur carrière : les impressions déclarées nous permettaient d'identifier des éléments saillants et de rupture dans l'évolution des parcours de formation ;

- L'évolution des savoirs à enseigner, des méthodes d'enseignement et des liens avec les autres champs disciplinaires, notamment techniques : ces éléments renvoyaient plus directement à l'évolution de l'épistémologie scolaire.

Nous avons ensuite retranscrit les entretiens et les avons analysés en distinguant les différentes facettes de l'épistémologie scolaire que chaque entretien nous permettait de déceler et avons identifié ce qui faisait entrer en résonance les différents éléments de mémoires individuelles que nous avons recueillies.

\footnotetext{
${ }^{5}$ Enseignement Agricole
} 
Ces éléments ont ensuite guidé notre recherche documentaire, afin de croiser les informations et de mieux comprendre le contexte de l'époque, à l'aide de documents d'archives ou d'enquêtes historiques. C'est ainsi que nous avons mobilisé différentes documents tels que les circulaires relatives au diplôme, les référentiels et les recommandations pédagogiques associées, les bulletins de l'INRAP ${ }^{6}$, les bulletins du BUP ${ }^{7}$, ou bien les revues scientifiques en didactique des sciences et techniques notamment Aster et Didaskalia, afin de comprendre l'évolution des sciences physiques et chimiques à l'Education Nationale et dans l'Enseignement Agricole.

Nous avons ensuite ordonné tous ces éléments en un récit unique, que nous avons soumis ensuite aux six interviewés ou inspecteurs interrogés, de façon à identifier les accords ou dissonances par rapport à leur expérience vécue.

Nous rendons compte de ce récit en distinguant deux parties : l'évolution des liens entre l'enseignement des sciences physiques et chimiques à l'éducation nationale et dans l'enseignement agricole, puis nous analysons les contenus de quelques curriculums de l'enseignement secondaire agricole (correspondant aux diplômes de niveau IV).

\section{L'évolution dans les manières d'enseigner et d'évaluer les sciences physiques et chimiques dans l'enseignement agricole, à l'ombre de l'éducation nationale}

La référence à l'éducation nationale occupe une place importante dans les discours des inspecteurs interrogés : l'éducation nationale est « la grande sœur, la maison mère, on ne pouvait pas rester complètement à l'écart » $\left(\mathrm{I}_{2}\right)$. L'éducation nationale («l'EN») est ainsi dans les discours l'institution qui contraint l'enseignement agricole à changer : « la première évolution, la disparition du BTA $^{8}$ était une demande de l'EN pour s'aligner sur leur forme de bac professionnel » $\left(\mathrm{I}_{5}\right)$, «les gros changements ont été la suppression du Bac D' et l'intégration au bac $\mathrm{S}$ avec une spécialité, et puis bien sûr récemment les BEPA ${ }^{9}$ et Bac Pro au lieu d'être en 4 ans le bac pro passe en 3 ans » $\left(\mathrm{I}_{2}\right)$. Mais l'éducation nationale est également l'institution par rapport à laquelle l'enseignement agricole peut se distinguer : « autrefois, l'enseignement agricole a été un peu révolutionnaire, c'est dans l'enseignement agricole qu'a été introduit les $\mathrm{CCF}^{10}(\ldots)$ ». De même, un inspecteur fait référence au rôle qu'ont joué les réflexions sur une réforme de l'enseignement scientifique à l'éducation nationale dans les années 70 : «le PSSC $^{11}$ a essaimé en France au niveau de la commission Lagarrigue et le ministère de l'agriculture a été convaincu qu'il fallait qu'on modernise notre enseignement en classe de seconde et qu'on s'inspire de tout ça et il fallait qu'on fasse une expérimentation. 12 établissements expérimentaux portés ensuite à 17 ont été financés » $\left(\mathrm{I}_{1}\right)$. Nous développons par conséquent dans cette partie l'évolution des liens entre l'enseignement agricole et l'éducation nationale, dans les réformes de l'enseignement des sciences physiques et chimiques.

\subsection{Les années 70 : le temps des réformes de l'enseignement des sciences physiques et chimiques à l'éducation nationale}

\subsubsection{Un contexte propice aux réformes}

\footnotetext{
${ }^{6}$ Institut National de Recherches et d'Applications Pédagogiques

${ }^{7}$ Bulletin de l'Union des Physiciens, édité par l'Union Des Professeurs de Physique et de Chimie

${ }^{8}$ Brevet de Technicien Agricole

${ }^{9}$ Brevet d'Etudes Professionnelles Agricoles

${ }^{10}$ Contrôle en Cours de Formation

${ }^{11}$ Physical Science Study Committee
} 
Plusieurs éléments font que s'engage dans les années 70 un mouvement important de remise à plat de l'enseignement des sciences physiques et chimiques à l'éducation nationale.

Tout d'abord, le contexte international s'inscrit dans une remise en cause du modèle traditionnel, abstrait, théorique et très mathématique, de l'enseignement des sciences. Ce mouvement international commence aux Etats-Unis en 1956, quand un groupe d'enseignants américains de lycée et d'université, encadré par des professeurs du MIT ${ }^{12}$, forme le Physical Science Study Committee (PSSC) dans le but de réfléchir à une réforme de l'enseignement de la physique et de la chimie : «en ce qui concerne la physique, les Etats Unis sont à l'avantgarde et l'action du Physical Science Study Committee (PSSC) mérite de retenir spécialement l'attention en raison de son ampleur, de ses effets et aussi parce qu'elle a commencé avant toute autre » $\left(\right.$ Unesco $^{13}, 1966$, p. 3). L'électrochoc reçu par la lancée de Spoutnik par les russes conduisit l'état fédéral à financer de façon très importante le projet PSSC afin d'améliorer l'enseignement des sciences. En une dizaine d'années, de nombreux matériels pédagogiques furent élaborés : manuels scolaires pour élèves, pour enseignants, films, travaux pratiques (fiches et matériels). L'approche prônée par le PSSC a d'abord été expérimentée dans les états de l'Ouest américain avant de se diffuser ensuite dans certains pays européens (Robin, 1968 ; OCDE ${ }^{14}$, 1965, Unesco, 1966). Des changements curriculaires se mettent en place dans les années 60, comme par exemple le HPP (Harvard Physical Project) dans certains états américains, les projets de la fondation Nuffield en Angleterre. L'approche suivie par ces changements est en rupture avec ce qui se fait en France à la même époque : il ne s'agit pas de présenter la physique comme une somme de connaissances, mais comme une activité. La méthode expérimentale, la recherche et la découverte ont donc une place centrale dans le dispositif : "ces projets sont caractérisés encore davantage par l'évolution des méthodes, par le souci de faire participer l'élève de façon active à la construction du savoir et développer chez lui une attitude scientifique. D'où la grande place accordée à la méthode de résolution de problèmes » (Host, 1980, p. 81).

Un deuxième élément de contexte joue un rôle important : la réforme des mathématiques dites «modernes » bouleverse l'enseignement traditionnel des mathématiques en donnant une grande importance à l'axiomatisation et aux structures mathématiques formelles dès l'école primaire. Cette abstraction plus poussée des mathématiques conduit à redessiner les rapports entre la physique et les mathématiques : «face aux mathématiques modernes promues dans les années 1960 comme une science déductive, et non comme une science expérimentale, mais critiquées pour leur formalisme et leur abstraction - celles-ci seraient trop éloignées du monde sensible des physiciens -, les sciences physiques et naturelles mettent en avant la place et le rôle de l'expérience, ainsi que le primat des méthodes sur les contenus, comme élément fondateur de leur identité pour affirmer leur légitimité » (D’Enfert, 2011, p. 14-15).

Ces éléments font qu'à l'orée des années 70 un important mouvement de réformes se met en place en France dans le but d'améliorer l'enseignement des sciences physiques et chimiques.

\subsubsection{Une commission ministérielle pour réformer l'enseignement des sciences physiques et chimiques}

En 1970, la Société Française de Physique (SFP) travaille en collaboration avec l'Union des Physiciens (UDP) et la Société Chimique de France (SCF), et obtient le soutien de l'Académie des Sciences pour demander une réforme de l'enseignement des sciences physiques et chimiques en France : « les programmes de physique de lère et de 2ème n'ont

\footnotetext{
${ }^{12}$ Massachusetts Institute of Technology

${ }^{13}$ Organisation des Nations unies pour l'éducation, la science et la culture

${ }^{14}$ Organisation de Coopération et de Développement Economiques
} 
pas changé depuis depuis 50 ans, et la Société Française de Physique en particulier est très sensibilisée à l'inadaptation totale des programmes actuels. Il nous semble un fait essentiel et pressé de réunir une commission ministérielle sur ce sujet » (président de la SFP, Jacques Friedel, cité par Hulin, 1991, p. 17).

M. Guichard, le ministre de l'EN de l'époque, accède à cette demande et crée la Commission ministérielle de rénovation de l'enseignement des sciences physiques, appelée plus communément «commission Lagarrigue» du nom de son premier président. Cette commission a alors pour objectif de réviser en profondeur l'enseignement des sciences physiques, chimiques et technologiques en France.

Cette commission est composée de membres de l'enseignement secondaire, supérieur, de l'Inspection Générale et de conseillers représentants des disciplines voisines ou bien extérieures au monde de l'enseignement (BUP, 1977).

L'esprit de la réforme suivi par la commission Lagarrigue met les travaux pratiques et la démarche expérimentale en premier plan : «l'importance qui a été accordée à l'approche expérimentale correspond à une option fondamentale de la Commission, sur laquelle celle-ci ne saurait revenir » (BUP, 1977, p. 45). Cette approche expérimentale doit de plus être associée à une pédagogie active des élèves : « un enseignement tel que nous le concevons nécessite un développement des travaux pratiques, organisés de façon moins scolaire avec davantage d'initiative donnée aux élèves ${ }^{15}$ (BUP, 1977, p. 42). La réforme que la commission propose consiste aussi en un travail sur le contenu à enseigner : " nous proposons d'abandonner un enseignement du type cloisonné entre les diverses parties de la Physique et de la Chimie (statique, dynamique, optique, électricité, chimie minérale, chimie organique) au profit d'un enseignement intégré », les thèmes et techniques (par exemple l'électronique) occupent une place importante.

La commission a conçu des avant-projets qui ont été expérimentés, pour le lycée d'abord dans 7 établissements de Paris et du Nord (1972-1973) puis dans 14 établissements de l'Académie de Grenoble (1973-1974) : « au total environ 100 professeurs et 2500 élèves ont été associés à cette opération »(BUP, 1977, p. 124). Pour les expérimentations du $1^{\text {er }}$ cycle (collège), un laboratoire de pédagogie «a entrepris une vaste expérience pédagogique d'enseignement modulé (axé sur des situations expérimentales concrètes) qui atteignait dans sa phase finale 15000 élèves par an » (BUP, 1977, p. 113).

\subsection{Le souffle réformateur s'étend à l'enseignement agricole}

\subsubsection{L'appel au changement}

Les travaux de la commission Lagarrigue ont laissé une empreinte importante dans l'enseignement agricole, à la fois par la volonté de placer la démarche expérimentale au centre de l'enseignement des sciences physiques et chimiques, mais aussi par la manière de préparer la réforme, via une période d'expérimentation dans des établissements scolaires. C'est ainsi que l'APEPA $^{16}$ alerte en septembre 1973 sur les conditions de l'enseignement des sciences physiques dans l'enseignement technique agricole : «le ministère n'a pas encore reconnu l'obligation de dédoublement des classes pour permettre de réaliser un enseignement expérimental. Or comment réaliser celui-ci avec des classes surchargées s'il n'est pas possible de les dédoubler? »(APEPA, 1973, p. 213). De même, Goudet (1974, p. 20) inscrit les travaux de la commission Lagarrigue dans la problématique propre à l'Enseignement Agricole : «parallèlement à un enseignement technologique agronomique dit professionnel,

\footnotetext{
${ }^{15}$ Issu du rapport sur la première évaluation du coût de la rénovation de l'enseignement des sciences physiques, janvier 1973

${ }^{16}$ Association des Professeurs de l'Enseignement Public Agricole
} 
l'enseignement agricole doit former des élèves capables d'accéder aux niveaux les plus élevés tant des professions agricoles, que des professions attachées aux sciences de la vie. Dans le cadre d'une telle option, la formation scientifique que nous assumerions devrait être de haut niveau et les problèmes soulevés par les caractères de la démarche scientifique, problèmes qui apparaissent clairement dans le projet Lagarrigue, ne peuvent nous laisser indifférents ». Comme l'explique $\mathrm{I}_{1}$, « on ne pouvait pas rester à la traîne d'un courant aussi important que celui de la commission Lagarrigue ».

\subsubsection{L'EA comme laboratoire}

La commission Lagarrigue finit ses travaux au moment ou la réforme Haby de 1975 (collège unique) se met en place. Toutefois son esprit perdure au sein de l'INRAP (Institut National de Recherches et d'Applications Pédagogiques), créé en 1966 à Dijon, dont le rôle était de proposer des stages de formation, mettre en œuvre des expérimentations pédagogiques, produire des ressources pédagogiques ou disciplinaires.

C'est dans cet institut que des expérimentations sur l'utilisation pédagogique de la méthode expérimentale en sciences physiques ont été faites (Goudet \& Coudray, 1976). Des lycées expérimentaux (d'abord 12 puis 17) ont ainsi mis en œuvre des nouvelles manières d'aborder l'expérience en sciences physiques et chimiques, du nouveau matériel et de nouvelles évaluations : «depuis 1975, des professeurs de 1'Enseignement Agricole Public contribuent à la rénovation de l'enseignement des sciences physiques. Leur objectif premier est de faire acquérir la méthode expérimentale à leurs élèves. Depuis 1977, des élèves de l'enseignement agricole public sont engagés dans l'expérimentation de la rénovation et des épreuves d'examen nouvelles ont été mises en place à la session de $1980 »$ (Goudet, 1981).

Ces expérimentations ont conduit « le ministère de l'agriculture à adopter les programmes de physique-chimie de l'éducation nationale (...) Cela a été le début de l'idée de parité entre l'EA et l'EN. Le travail fait a été repris sur d'autres programmes de l'EA et dans la foulée on a réécrit et expérimenté tous les programmes de BTA $\gg\left(\mathrm{I}_{1}\right)$.

Cet élan de rénovation de l'enseignement des sciences physiques et chimiques s'est ainsi poursuivi en réinvestissant les travaux sur l'évaluation du groupe Chapham, issu de la commission Lagarrigue (Goudet, 1981). Les lycées expérimentaux ont en effet mis en œuvre les 4 types d'exercice proposés par le groupe Chapham : des questions sur les méthodes et raisonnement expérimentaux ; un problème de physique ; des questions à réponse courte ; une lecture critique d'un texte scientifique (Groupe Chapham, 1980, p. 39). Selon $\mathrm{I}_{1}$, « quand on a généralisé cette structure a un peu éclaté, car quatre types d'évaluation, c'était compliqué à mettre en place, mais il en est resté quelque chose : des évaluations qui s'appuient sur des documents, des expériences ou bien des faits de la vie quotidienne ». L'analyse faite des épreuves de sciences physiques (BTA et Bac D' de 1980, Goudet, 1981) montre que les dispositions suivantes ont été retenues pour faire évoluer les épreuves : pour l'épreuve écrite, «les questions doivent reposer sur une exploitation de données telles que : résultats expérimentaux se présentant sous forme de tableau de valeurs, graphiques, enregistrements de trajectoires pour la mécanique, etc., fiches techniques d'appareils, dossiers d'informations et compte rendus d'expériences ou d'observations; une des questions peut être une interprétation de faits de la vie quotidienne, ou de faits techniques simples »; pour l'épreuve oral de contrôle, « [l'oral] se déroule au laboratoire et permet soit de réaliser une manipulation simple par le candidat, qui la présentera et l'interprétera, soit d'interroger le candidat sur une expérience réalisée par l'examinateur » (Goudet, 1981). Cette manière d'évaluer fait dire à $\mathrm{I}_{4}$ que « le BTA était un diplôme sensationnel (...) cette façon d'aborder l'épreuve par les textes a complètement disparu lors de la réforme du bac pro, bac techno ». 
Si l'on observe bien une évolution des épreuves vers une place plus grande apportée à l'expérimentation, il reste que cette évolution est contenue dans le cadre d'une épreuve terminale écrite : "dans le cadre de cette expérimentation sur les 17 établissements on avait proposé de faire évoluer en conséquences les sujets d'examen, mais on n'était pas encore mûrs pour introduire des contrôles expérimentaux $\gg\left(\mathrm{I}_{1}\right)$.

C'est alors l'introduction des contrôles en cours de formation (CCF) qui a conduit à la mise en place d'une épreuve pratique : «le CCF est apparu dans les années 80, il y a eu aussi une expérimentation avec 2 fois 20 établissements, et entre autres la grande innovation ça a été l'introduction des CCF. Les CCF nous ont permis d'introduire les contrôles expérimentaux. L'EN a suivi le même cheminement quelques années plus tard. L'EN a laissé l'EA être un laboratoire, c'est comme ça que j'interprète l'histoire » $\left(\mathrm{I}_{1}\right)$. De même pour $\mathrm{I}_{2}$, « la grande révolution, ça a été ça : les $\mathrm{CCF} »$.

En effet, les épreuves pratiques sont introduites dans la rénovation des formations agricoles de 1985 (création des baccalauréats professionnels) et il faut attendre la rentrée 1996 pour qu'elles le soient à l'éducation nationale : «l'enseignement des sciences physiques a été profondément rénové dans les sections de baccalauréat professionnel, depuis la rentrée scolaire 1996. (...) La nécessité d'une approche concrète des questions posées est affirmée ; elle se traduit par la place accordée aux activités expérimentales réalisées par les élèves en travaux pratiques et en classe laboratoire. A compter de la session 1998, les compétences expérimentales des candidats seront reconnues à l'examen grâce à la mise en place d'une épreuve pratique » (Secrétan, 1998).

Ce décalage dans la prise en compte de l'expérimentation en épreuve pratique de baccalauréat participe (avec les contrôles en cours de formation et la pluridisciplinarité) à qualifier l'enseignement agricole de « laboratoire de l'EN » $\left(\mathrm{I}_{1}, \mathrm{I}_{2}\right)$.

\subsection{Les réformes à l'EN qui impactent à nouveau l'EA}

Après une période d'innovation dans l'enseignement agricole, c'est de l'éducation nationale que proviennent les principaux changements à partir de la fin des années 2000. C'est par exemple le cas de la démarche d'investigation, qui, en tant que nouvelle modalité pédagogique dans l'enseignement des sciences, se retrouve promue dans les réformes successives (depuis le plan de rénovation de l'enseignement des sciences et de la technologie à l'école en 2000, la réforme du collège en 2006, celle du lycée général et technique en 2010). Cette démarche est également préconisée dans l'enseignement agricole à partir de 2010 (dans le document d'accompagnement du module MG4 du baccalauréat professionnel) et se généralise alors à l'ensemble des niveaux et filières agricoles.

L'innovation majeure concerne toutefois les conséquences de l'adoption du « socle commun de connaissances et de compétences » dans la loi d'orientation et de programme pour l'avenir de l'école du 23 avril 2005, qui introduit un nouveau cycle de réformes à l'éducation nationale et qui structure autour de la notion de compétences toutes les écritures des nouveaux programmes de scolarité qui se succèdent $\left(\mathrm{I}_{6}\right)$.

Cette approche se diffuse rapidement à tous les niveaux dans les prescriptions officielles de l'EN (jusqu'à devenir une modalité d'attribution du diplôme du brevet des collèges en 2010), et aucune discipline, ni niveau n'est tenu à l'écart de cette propagation.

L'approche par compétences redéfinit les orientations scolaires, non par le contenu en termes de connaissances disciplinaires, mais par des objectifs généraux visés (des compétences transversales et transférables). Ces objectifs sont déclinés en tâches, méthodes ou comportements et des critères d'évaluation sont précisés. La modalité pédagogique privilégiée est le travail actif et autonome des élèves par tâche complexe. 
Cette rapide conversion de l'éducation nationale se fait dans un contexte international où les directives européennes incitent à la mise en place d'un cadre européen de certification de compétences dans une société devenue «société de la connaissance » (conseil européen de Lisbonne, 2000). Le choix opéré par le programme de l'OCDE Pisa d'évaluer les compétences des jeunes de 15 ans afin de comparer les acquis d'élèves ne suivant pas les mêmes programmes contraint de plus les politiques éducatives à adhérer à cette approche.

L'enseignement agricole a donc suivi ce mouvement général, avec le retard que l'on peut attribuer à son autonomie par rapport à l'éducation nationale $\left(\mathrm{I}_{6}\right)$. Alors que cette dernière s'est mise en ordre de marche dès la rentrée 2006, c'est avec la rénovation de la voie professionnelle que l'enseignement agricole va vraiment rentrer dans ce mouvement, à partir de la rentrée 2010. Ce passage à la logique des compétences va se retranscrire tout d'abord par la manière d'écrire les référentiels de formation : une place centrale est accordée aux capacités plutôt qu'aux savoirs à enseigner, «l'écriture des référentiels ne se limitent plus à un horizon de savoirs à transmettre, mais vise à servir des objectifs de formation. Désormais, tous les référentiels de l'enseignement agricole sont articulés sur les capacités, les contenus viennent ensuite, dans les documents d'accompagnement $\gg$ (DGER, 2010, p. 140). Ce passage des savoirs vers les capacités tient à la place primordiale occupée maintenant par le référentiel de certification: "l'écriture des référentiels de diplôme a été réalisée selon une nouvelle méthodologie qui donne une place centrale au référentiel de certification élaboré avec une liste structurée de capacités à partir du référentiel professionnel »(DGER, 2010, p. 122). Ce sont alors les situations professionnelles significatives (SPS), décrites dans les référentiels de certification, qui déterminent les capacités à certifier.

Comme toutes les disciplines de l'enseignement agricole, l'enseignement de la PhysiqueChimie dans un contexte mouvant de liens entre savoirs savants et savoirs à enseigner change, à la fois dans les manières d'enseigner que dans les manières d'évaluer.

\subsection{Les sciences physiques et chimiques dans l'enseignement agricole : un lien fort avec l'éducation nationale}

Cette description montre que les sciences physiques et chimiques dans l'enseignement agricole sont une discipline à cheval entre deux institutions. En effet, « on ne peut jamais se différencier complètement de l'éducation nationale. Donc chaque fois qu'il y a des nouveaux programmes qui apparaissent à l'EN, immédiatement ça déteint sur l'agriculture » $\left(\mathrm{I}_{2}\right)$. Mais dans le même temps, cet enseignement s'affranchit de cette dépendance, notamment par la spécificité des savoirs enseignés dans les filières professionnelles : «on ne pouvait pas être complètement déconnecté de l'EN, surtout dans les filières générales. Dans les filières professionnelles, alors là c'était pas du tout pareil, tu faisais une physique-chimie très appliquée dans les BTA, les viti-oeno, le machinisme (...) là c'est vrai qu'on avait beaucoup plus de liberté et les programmes étaient beaucoup plus axés sur le devenir des élèves et leur orientation professionnelle» $\left(\mathrm{I}_{2}\right)$. Tous les témoignages soulignent l'importance de l'introduction des CCF (notamment l'épreuve pratique) et de la pluridisciplinarité dans les référentiels de l'enseignement agricole, mis en application bien en amont des pratiques de l'éducation nationale, ce qui justifie l'idée que l'enseignement agricole ait pu jouer le rôle de « laboratoire » pour l'enseignement des sciences physiques et chimiques.

\section{La place des sciences physiques et chimiques dans les référentiels de l'enseignement secondaire agricole}

Nous nous intéressons maintenant aux contenus des référentiels de formation, car les témoignages mettent l'accent sur les spécificités de certains savoirs à enseigner, comme par 
exemple $\mathrm{I}_{2}$ qui déclare : «quand nous étions en formation, par exemple, on avait 2 types de cours, en physique sur tout ce qui portait sur la mécanique, on étudiait les moteurs à explosion, que ce soit les moteurs classiques alternes, les moteurs diesel, les moteurs à réaction, il y avait une grande partie sur les transmissions, parce qu'on y enseignait ça à l'enseignement agricole et ce n'était pas le cas à l'éducation nationale. Et en chimie, notre originalité, c'était les biomolécules, la biochimie (...) Voila une différence notoire, on avait même des cours de chimie du sol, de pédologie ».

Nous présentons tout d'abord l'évolution des diplômes et nous détaillons la place et les contenus de l'enseignement des sciences physiques et chimiques à travers l'analyse de quelques référentiels de formation de niveau IV depuis 1985 dans les filières les plus représentatives de l'enseignement agricole.

\subsection{Quelques éléments sur l'évolution des diplômes de l'enseignement secondaire agricole}

La loi du 2 août 1960 sur l'enseignement agricole et la formation professionnelle agricole conduit à la transformation des écoles pratiques d'agriculture et des écoles ménagères agricoles en collèges agricoles, et à la création de lycées agricoles.

A partir de 1968, deux cycles sont mis en œuvre : l'enseignement technique agricole court (qui délivre le $\mathrm{BAA}^{17}$, le $\mathrm{CAPA}^{18}$ puis le $\mathrm{BEPA}^{19}$ à partir de 1971) dans les collèges agricoles, appelés à partir de 1979 lycées d'enseignement professionnel agricole, et l'enseignement technique long (qui délivre le BTA et le Bac D') dans les lycées agricoles (Lelorrain, 2005).

L'ensemble des formations est rénové suite aux lois de 1984. C'est tout d'abord le BTA qui est rénové en 1985, puis les CAPA et BEPA en 1989. Selon Lelorrain (2005, p. 73), « dès sa réorganisation, l'existence du BTA est remise en question par l'institution à l'éducation nationale, en 1985-1986, de deux nouveaux diplômes, le baccalauréat technologique et le baccalauréat professionnel ». C'est pourquoi sont créés en 1993, deux baccalauréats technologiques agricoles (le $\mathrm{STAE}^{20}$ et le $\mathrm{STPA}^{21}$ ) et en 1995, les baccalauréats professionnels agricoles, qui remplacent progressivement les BTA.

Nous nous intéressons par la suite à la place des sciences physiques et chimiques dans les diplômes les plus représentatifs de l'enseignement secondaire agricole de cycle long: les BTA, les baccalauréats technologiques et professionnels. Nous avons analysé les référentiels en nous focalisant sur les savoirs qui étaient les plus cités lors des entretiens, comme la chimie du sol, les biomolécules, l'approche énergétique en physique, les moteurs électriques, etc.

\subsection{Le Brevet de Technicien Agricole (BTA)}

\subsubsection{Architecture et horaires}

La rénovation du BTA en 1984 met en place des innovations pédagogiques importantes : les disciplines sont regroupées en modules et le référentiel de formation ne précise pas les frontières disciplinaires, une grande partie du diplôme s'obtient par des «contrôles certificatifs en cours de formation»(CCF). Trois classes de modules sont définies: les secteurs de base (où l'on retrouve les enseignements généraux: français, langues, mathématiques, histoire-géographie), les secteurs professionnels (où l'on retrouve les

\footnotetext{
${ }^{17}$ Brevet d'Apprentissage Agricole

${ }^{18}$ Certificat d'Aptitude Professionnelle Agricole

${ }^{19}$ Brevet d'Etudes Professionnelles Agricoles

${ }^{20}$ Sciences et Techniques de l'Agronomie et de l'Environnement

${ }^{21}$ Sciences et Technologiques du Produit Alimentaire
} 
enseignements scientifiques) et les secteurs de qualification professionnelle (où l'on retrouve les modules professionnels spécifiques à la filière). Les programmes sont écrits sous la forme de capacités ( «être capable de ») pour les secteurs de base et qualification, et sous la forme de verbes à l'infinitif (définir, montrer, décrire, classer, utiliser...) pour les modules de secteur.

Les programmes regroupent deux années d'enseignement et on trouve les sciences physiques et chimiques :

- Dans les modules de secteur de base : 6 heures pour des supports de texte en philosophie (module B5) et $18 \mathrm{~h}$ avec les mathématiques et traitement de données numériques et graphiques (module B6) «dont les domaines supports pourront être l'électricité, la pHmétrie ».

- Dans le module de secteur 1 intitulé « connaissance de la matière et du vivant », qui comportait de $45 \mathrm{~h}$ de sciences physiques et chimiques dans la filière commercialisation et services ( $\mathrm{SC} 1)$, à $85 \mathrm{~h}$ dans les options production (SP1) et transformation (ST1). Ce module est commun avec la biologie (pour les mêmes horaires) et comprend en 1985 de la philosophie (10h).

- Dans les modules de qualification professionnelle : le nombre d'heures varie suivant la qualification, de 0 (par exemple en animalerie de laboratoire) jusqu'à $105 \mathrm{~h}$ en machinisme.

L'objectif général pour les sciences physiques et chimiques est la «priorité de préférer un nombre limité de concepts scientifiques de base à la non-assimilation d'un grand nombre d'idées vagues et fluides ».

Une rénovation de ce diplôme a lieu en 1992, sans changement sur les contenus, seules les 10 heures communes avec la philosophie n'apparaissent plus.

On résume dans le tableau $\mathrm{n}^{\circ} 1$ la répartition horaire de l'enseignement des sciences physiques et chimiques ramené à l'ensemble du référentiel :

\begin{tabular}{|c|c|c|c|}
\hline BTA & $\begin{array}{l}\text { Sciences physiques } \\
\text { et chimiques }\end{array}$ & $\begin{array}{l}\text { Total référentiel } \\
\text { (hors stages) }\end{array}$ & $\begin{array}{c}\% \text { de Sciences } \\
\text { physiques et chimiques }\end{array}$ \\
\hline 1985 & $60 \mathrm{~h}$ à $215 \mathrm{~h}$ & $1800 \mathrm{~h}$ à $1880 \mathrm{~h}$ & $3.3 \%$ à $11.4 \%$ \\
\hline 1992 & $55 \mathrm{~h}$ à $215 \mathrm{~h}$ & $1895 \mathrm{~h}$ à $2005 \mathrm{~h}$ & $3 \%$ à $11 \%$ \\
\hline
\end{tabular}

Tableau $n^{\circ} 1$ : répartition horaire des sciences physiques et chimiques dans le BTA

\subsubsection{Les savoirs à enseigner et les recommandations pédagogiques}

L'écriture modulaire des référentiels de 1985 et 1992 du BTA ne réfèrent pas les savoirs à enseigner à des disciplines spécifiques : les modules SP1 et ST1 sont par exemple communs aux sciences physiques et chimiques et à la biologie, mais la répartition des savoirs à l'intérieur du module n'est pas explicitée. Cette volonté d'intégrer les disciplines autour de savoirs particuliers se retrouve dans le titre du module professionnel QP1 qui concerne les «sciences et techniques communes aux productions végétales », «il doit conduire à des harmonisations de vocabulaire impératives » (recommandations pédagogiques de 1985). On y trouve ainsi des savoirs relatifs à la chimie du sol (détermination chimique des engrais, identification de la nature du sol «à mettre en place avec le professeur de phytotechnie ») et du vivant (biomolécules) : un des objectifs est ainsi de «décrire la structure, exposer les propriétés et les rôles des principales molécules organiques caractéristiques du vivant». De même, l'énergie chimique est abordée et « la liaison avec la biologie est ici indispensable ».

Le module de secteur 1 s'articule ainsi à la biologie, mais renvoie également à d'autres modules : l'étude de l'énergie mécanique doit «nécessairement déboucher sur l'étude de la liaison tracteur-outil, l'analyse de documents techniques », l'énergie thermique est «un concept essentiel qui concerne aussi bien l'introduction à l'énergie chimique, que la climatologie du module QP1 ou les moteurs thermiques du module QP 4, les transformations 
dans le progrès industriels dans le module ST2. Il convient donc de choisir les supports expérimentaux et les manipulations se rapportant aux modules concernés ».

Si les recommandations de 1985 insistent pour que le contenu des modules de secteur constituent les bases qui seront approfondies, exemplifiées, voire supports d'expérimentation dans les modules de qualification, les recommandations de 1992 ne mentionnent plus ce lien : les recommandations sont uniquement axées sur les efforts à faire dans la liaison à l'intérieur du module : le lien avec la biologie doit être renforcé, notamment dans l'étude de l'énergie chimique et rayonnante, l'objectif 1 du module est alors de "montrer l'unité chimique de la matière vivante (composition, fonctions, réactions) au travers de sa diversité (structures et rôles) ».

On note également dans ces nouvelles recommandations la présence d'éléments de physique moderne : l'énergie nucléaire, les relations d'Einstein, l'effet photoélectrique, la dualité onde corpuscule qu'il est préconisé d'approcher via une étude historique. De même, le module QP4 aborde les moteurs triphasés, l'électronique (la jonction P-N, les semi-conducteurs et les transistors, dont l'étude sera enlevée lors de la rénovation de 1992).

$\mathrm{Du}$ point de vue des méthodes pédagogiques, la référence aux travaux pratiques est très présente et associée à des savoirs particuliers : « «pour tous les élèves, [l'enseignement des propriétés acido-basiques] doit donner lieu à des travaux pratiques (dosages, courbes de neutralisation » (recommandations 1992), «la pratique de la méthode expérimentale pour acquérir les concepts de redox est indispensable », «observations, analyses, branchements de matériel, etc. sont des activités pédagogiques à mettre en œuvre » en électricité.

En 1992, une recommandation générique apparait sans explicitation : "la pratique de la méthode expérimentale dans son intégralité constitue la méthode pédagogique à privilégier ».

\subsection{Les Baccalauréats Technologiques STAE et STPA}

\subsubsection{Architecture et horaires}

En 1993, les deux filières technologiques STAE (Sciences et Techniques de l'Agronomie et de l'Environnement) et STPA (Sciences et Technologies du Produit Alimentaire) sont mises en place et deux baccalauréats technologiques sont créés. Les principes pédagogiques poursuivis reprennent les principaux éléments de la reforme de 1984 (modules pluridisciplinaires, CCF). La formation se déroule autour de dix matières (au sens de regroupement de plusieurs disciplines). Le référentiel de formation distingue les programmes de première et de terminale.

En STAE, les sciences physiques et chimiques sont :

- dans la matière M7 (« la matière et le vivant »), qui est commune à la physique, la chimie, la biologie et l'écologie ;

- dans la matière M10-4 : 20h en technologies des aménagements en 1993 puis 10h en 2002.

En STPA, les sciences physiques et chimiques sont :

- dans la matière M7 (« la matière et le vivant»), qui est commune à la physique, la chimie, la biologie et l'écologie ;

- dans la matière M9 (physique appliquée et technologies alimentaires) en 1993, en commun avec la biologie, la microbiologie et les technologies alimentaires, puis dans la matière M8 lors de la rénovation de 2002 (sciences appliquées aux biotechnologies et à l'analyse des produits agroalimentaires), en commun avec la biochimie, la microbiologie et les technologies alimentaires. On trouvera les répartitions horaires et leur évolution dans les tableaux 2 et 3 :

\begin{tabular}{|c|l|l|c|}
\hline STAE & $\begin{array}{l}\text { Sciences physiques } \\
\text { et chimiques }\end{array}$ & $\begin{array}{l}\text { Total référentiel } \\
\text { (hors stages) }\end{array}$ & $\begin{array}{c}\text { \% de Sciences physiques } \\
\text { et chimiques }\end{array}$ \\
\hline
\end{tabular}




\begin{tabular}{|c|c|c|c|}
\hline 1993 & $192 \mathrm{~h}$ à $212 \mathrm{~h}$ & $1984 \mathrm{~h}$ & $9.7 \%$ à $10.7 \%$ \\
\hline 2002 & $180 \mathrm{~h}$ à $190 \mathrm{~h}$ & $1920 \mathrm{~h}$ & $9.4 \%$ \\
\hline
\end{tabular}

Tableau $n^{\circ} 2$ : répartition horaire des sciences physiques et chimiques dans le STAE

\begin{tabular}{|c|c|c|c|}
\hline STPA & $\begin{array}{l}\text { Sciences physiques } \\
\text { et chimiques }\end{array}$ & $\begin{array}{l}\text { Total référentiel } \\
\text { (hors stages) }\end{array}$ & $\begin{array}{c}\text { \% de Sciences physiques } \\
\text { et chimiques }\end{array}$ \\
\hline 1993 & $420 \mathrm{~h}$ & $1984 \mathrm{~h}$ & $21 \%$ \\
\hline 2002 & $300 \mathrm{~h}$ & $1920 \mathrm{~h}$ & $15.6 \%$ \\
\hline
\end{tabular}

Tableau $n^{\circ} 3$ : répartition horaire des sciences physiques et chimiques dans le STPA

\subsubsection{Les savoirs à enseigner et les recommandations pédagogiques}

L'objectif général de ces baccalauréats évolue avec la rénovation de 2002. En effet, l'objectif de 1993 de la classe de terminale fait de la culture scientifique un moyen de réussite scolaire : «acquérir une culture scientifique cohérente, dans le domaine des sciences physiques et biologiques, en vue de la poursuite d'études dans un secteur technologique », alors qu'elle devient un objectif en soi en 2002 : le module «contribue au développement d'une culture scientifique cohérente chez les élèves ».

En STAE, la matière M7 est commune à la biologie et aux sciences physiques et chimiques, mais les points de contact entre les deux disciplines sont rares, d'autant plus que les savoirs sont clairement séparés par leur discipline d'appartenance.

L'objectif 1 est commun aux deux disciplines : «mettre en évidence l'unité de la matière vivante et réaliser une approche concrète des biomolécules », mais il y a peu de synergie explicitée, à part pour l'étude des lipides où il est écrit : «interface avec la biologie : utilisation de documents ». De même pour l'étude de l'oxydoréduction : il s'agit de " donner des exemples de couples d'intérêt biologique ». Seule l'énergie chimique renvoie explicitement à la biologie : «cette partie relève du programme de chimie mais doit être traitée en concertation étroite avec l'enseignant de biologie ». Il n'y a pas d'horaires affectés à la pluridisciplinarité.

Des éléments de physique moderne sont dans les programmes : l'énergie nucléaire, dont l'objectif est «de sensibiliser le public au fait social que constitue l'utilisation de l'énergie nucléaire », le photon et la dualité ondes-corpuscules.

Entre 1992 et 2003, des savoirs disparaissent des programmes : l'électromagnétisme et le moteur asynchrone triphasé ne sont plus étudiés à partir de 2003.

Du point de vue des méthodes pédagogiques, il s'agit de « privilégier les approches concrètes, pratiques, expérimentales »(1992), et les travaux pratiques sont clairement identifiés et référencés. En 2002, « les méthodes privilégiées sont celles qui mettent l'élève en situation de construction active de ces connaissances dans les limites du possible », la moitié de l'horaire est en demi-groupe (un quart en TP, un quart en TD).

En STPA, les contenus sont les mêmes qu'en STAE, à quelques différences près : la réforme de 2002 conserve l'étude de l'électromagnétisme, mais enlève celle du moteur asynchrone triphasé (M9); l'étude des biomolécules se fait sans lien explicite avec la biologie et la biochimie qui sont pourtant dans le même module (M8).

\subsection{Le Baccalauréat technologique STAV (sciences et technologiques de l'agronomie et du vivant : agronomie - alimentation - environnement - territoires).}

\subsubsection{Architecture et horaires}

En 2006, les baccalauréats STAE et STPA fusionnent pour donner naissance au baccalauréat STAV. 
Les sciences physiques et chimiques sont dans la matière M9 «matière et énergie dans les systèmes » $(74 \mathrm{~h}$ de physique, $81 \mathrm{~h}$ de chimie et $3 \mathrm{~h}$ de pluridisciplinarité avec les sciences et techniques des équipements - STE) et intervient dans la matière M7 «le fait alimentaire » pour $27 \mathrm{~h}$ de pluridisciplinarité avec la biologie, les sciences et technologies agronomiques et des équipements.

En 2013, une rénovation de ce diplôme fait apparaître les sciences physiques et chimiques seules en tant que matière d'enseignement technologique, la matière M8 («mobiliser des savoirs et des savoir-faire scientifiques afin d'appréhender les processus du vivant et de comprendre les enjeux énergétiques »), avec un horaire de $139 \mathrm{~h} 30$, dont $16 \mathrm{~h}$ de pluridisciplinarité avec les STE). Les sciences physiques et chimiques apparaissent également dans deux matières professionnelles à l'initiative de l'établissement (M9 pour 31h) : transformation alimentaire et sciences et technologies des équipements (en pluridisciplinarité avec les STE) et la possibilité d'une pluridisciplinarité dans la matière M7-1 («le fait alimentaire ») pour $12 \mathrm{~h}$ avec la biologie-écologie.

\begin{tabular}{|c|c|c|c|}
\hline STAV & $\begin{array}{l}\text { Sciences physiques } \\
\text { et chimiques }\end{array}$ & $\begin{array}{l}\text { Total référentiel } \\
\text { (hors stages) }\end{array}$ & $\begin{array}{c}\text { \% de Sciences physiques } \\
\text { et chimiques }\end{array}$ \\
\hline 2006 & $185 \mathrm{~h}$ & $1860 \mathrm{~h}$ & $9.9 \%$ \\
\hline 2013 & $155.5 \mathrm{~h}$ à $188.5 \mathrm{~h}$ & $1922 \mathrm{~h}$ & $8.1 \%$ à $9.8 \%$ \\
\hline
\end{tabular}

Tableau $n^{\circ} 4$ : répartition horaire des sciences physiques et chimiques dans le STAV

\subsubsection{Les savoirs à enseigner et les recommandations pédagogiques}

Le programme du STAV est un programme sur deux ans, qui ne distingue pas l'année de première et celle de terminale.

Les sciences physiques et chimiques n'interviennent plus en binôme avec la biologie comme dans le STAE ou STPA, la matière M9 est en effet commune avec les sciences et techniques des équipements. Les savoirs référés aux deux disciplines sont clairement distingués dans le référentiel et une plage de pluridisciplinarité est prévue (3h). Les domaines qui établissaient des liens avec la biologie disparaissent: l'énergie chimique n'est plus un objet d'enseignement, les biomolécules font maintenant référence à l'alimentation plutôt qu'au vivant et leur étude chimique est isolée de la matière M7 consacré au « fait alimentaire ».

L'accent est mis sur l'enseignement expérimental «dû à un échange permanent entre observation et théorie qui se nourrissent l'un l'autre». «La curiosité et le questionnement doivent être suscités et trouvent réponse dans l'acquisition progressive de capacités relatives à l'analyse, l'observation, à l'émission d'hypothèses, à l'expérimentation, à la vérification et à la pratique de l'esprit critique ». La démarche expérimentale est ainsi explicitée dans ce référentiel. Les savoirs donnant lieu à des travaux pratiques sont clairement identifiés.

La notion de «risque chimique pour les utilisateurs mais aussi pour l'environnement» apparaît : il s'agit donc ici d'un nouvel objet d'enseignement. L'étude chimique du sol n'est pas abordée, de même que l'électromagnétisme et les moteurs. Le nucléaire disparaît aussi du programme.

Des éléments de physique moderne sont encore présents (le photon), mais la préconisation d'une approche historique disparaît.

Le référentiel de 2013 est très succinct et n'est plus décliné par année d'enseignement comme en 2006. Il faut se reporter aux documents d'accompagnement pour avoir une lecture plus approfondie des savoirs mis en jeu. Les objectifs de formation se font par entrées thématiques (l'alimentation, le contrôle qualité, les transports, le stockage et la production d'énergie par exemple) et non par la catégorisation classique des objets de la physique (électricité, mécanique, chimie organique, etc.). 
Si la démarche expérimentale est comme en 2006 posée comme fondamentale, celle-ci est explicitée en 2013 : l'enseignement de les sciences physiques et chimiques « doit permettre d'initier l'élève à la démarche scientifique c'est-à-dire de le rendre capable de mettre en œuvre un raisonnement pour identifier un problème, formuler des hypothèses, les confronter aux constats expérimentaux et exercer son esprit critique. Quand elle est possible la démarche d'investigation s'inscrit dans cette logique. L'approche expérimentale est donc à privilégier ». Ainsi, l'identification d'un problème apparaît comme un élément de la démarche scientifique à partir de la rénovation de 2013.

L'énergie nucléaire revient dans le référentiel, avec comme objectif d'《éclairer les élèves » en traitant les enjeux scientifiques d'une question médiatique et controversée. L'angle sous lequel est abordée l'énergie est celui du développement durable puisqu'un des objectifs est : « enjeux énergétiques dans une perspective de développement durable ».

Le lien avec les sciences et techniques des agroéquipements est renforcé avec une plage horaire de pluridisciplinarité de 16h sur le M8. Toutefois, aucun lien n'est explicité avec la biologie, malgré l'étude des biomolécules et l'objectif général accordé à l'«étude des processus chimiques liés au vivant $»$.

\subsection{Les Baccalauréats professionnels}

\subsubsection{Architecture et horaires}

Les classes de BTA disparaissent progressivement à partir de la rentrée 1996, au profit des baccalauréats professionnels.

La formation est structurée en modules d'enseignements généraux (MG 1 à 4), professionnels communs à tous les bacs pro (MP1 à 4) et spécifiques à la spécialité professionnelle. Les sciences physiques et chimiques se retrouvent isolées dans le module professionnel MP3 (Eléments de chimie du vivant, du sol et de l'environnement, Energétique), aucun horaire de pluridisciplinarité n'est prévu bien qu'il soit recommandé de mener des actions en ce sens. Lors de la rénovation de 2008, il n'y a pas eu de changement sur les contenus, mais une réduction d'horaire de cinq heures.

Avec la rénovation de la voie professionnelle (RVP), le diplôme est structuré autour d'un ensemble de modules d'enseignement généraux (MG 1 à 4) et de modules professionnels spécifiques à la filière. L'enseignement des sciences physiques et chimiques passe alors dans les modules d'enseignement général (le MG4) intitulé "culture scientifique et technologique », aux côtés des mathématiques, de l'informatique et de la biologie-écologie, à raison de $70 \mathrm{~h}$ sur 2 ans. Les sciences physiques et chimiques interviennent également dans des modules professionnels dans le tiers des baccalauréats professionnels, quelquefois de façon significative comme dans les filières laboratoire-contrôle-qualité (LCQ) (360h) ou agroéquipement (AEQ) (226h).

\begin{tabular}{|c|c|c|c|}
\hline Bac Pro & $\begin{array}{l}\text { Sciences physiques } \\
\text { et chimiques }\end{array}$ & $\begin{array}{l}\text { Total référentiel } \\
\text { (hors stages) }\end{array}$ & $\begin{array}{c}\text { \% de Sciences physiques } \\
\text { et chimiques }\end{array}$ \\
\hline 1996 & $75 \mathrm{~h}$ à $175 \mathrm{~h}$ & $1610 \mathrm{~h}$ & $4.6 \%$ à $11 \%$ \\
\hline 2009 & $70 \mathrm{~h}$ à $360 \mathrm{~h}$ & $1680 \mathrm{~h}$ & $4.2 \%$ à $21.4 \%$ \\
\hline
\end{tabular}

Tableau $n^{\circ} 5$ : répartition horaire des sciences physiques et chimiques dans le baccalauréat professionnel

\subsubsection{Les savoirs à enseigner et les recommandations pédagogiques}

Les référentiels de 1996 et 2008 sont identiques au point de vue des savoirs et méthodes à enseigner. 
Les sciences physiques et chimiques sont seules dans le module MP3 mais l'accent est mis sur le lien à construire avec les disciplines scientifiques et techniques : «selon les souhaits du conseil national des programmes sur l'enseignement des sciences expérimentales, celles-ci et les sciences techniques ne doivent pas s'opposer et se démarquer mais plutôt se compléter et se féconder mutuellement. Pour cette raison, il est fortement recommandé que des actions pluridisciplinaires avec les enseignants de biologie et ceux des enseignements techniques soient mises en œuvre pour traiter certains points du contenu de ce module ». L'objectif $1 \mathrm{du}$ MP3 consiste ainsi à «expliquer les phénomènes chimiques liés au sol et à l'environnement »: la chimie du sol est alors l'occasion de mettre en relation les sciences physiques et chimiques et l'agronomie. Les moteurs triphasés permettent un contact entre les sciences physiques et chimiques et les sciences et techniques des équipements, l'étude du photon et de l'effet photoélectrique est associée à la photosynthèse.

La méthode scientifique est à la fois une modalité pédagogique et un objet d'enseignement : « chaque fois que cela est possible on fera acquérir des éléments de la méthode scientifique : observer, analyser, émettre des hypothèses, expérimenter, critiquer, vérifier. L'enseignement de ce module doit donc réserver une grande place aux pratiques de laboratoire ».

La rénovation de la voie professionnelle (RVP) bouleverse les référentiels à partir de 2011.

Les sciences physiques et chimiques sont dans un module commun à la biologie, l'écologie, les mathématiques et l'informatique, mais aucune pluridisciplinarité n'est prévue ni même préconisée. Le seul lien opéré est le renvoi à la filière professionnelle : «on s'efforcera de contextualiser les supports de formation en fonction des secteurs professionnels et de s'appuyer sur des situations et exemples concrets ». La capacité à certifier est une capacité " générale »: «expliquer les faits scientifiques à l'aide des outils et des raisonnements de la physique et de la chimie » et le module de formation relève d'une «culture générale et technologique », qui consiste à «mobiliser des savoirs et utiliser des démarches scientifiques pour analyser, interpréter et utiliser des informations liées aux propriétés de l'eau, des solutions aqueuses, des biomolécules, de quelques systèmes mécaniques en équilibre et de certaines formes d'énergies ». La méthode scientifique devient un objet d'enseignement, au même titre que les savoirs scientifiques : "il est essentiel d'entraîner les apprenants à l'activité scientifique et de promouvoir l'acquisition de méthodes et de capacités. Les connaissances sont amenées conjointement au moment de cette acquisition ».

Toutefois, ce sont les savoirs scientifiques qui sont détaillés, plus que la nature de la méthode scientifique. Ainsi, les biomolécules, appliquées à l'alimentation, apparaissent dans le référentiel de 2011, tandis que la chimie du sol disparait. De même, en physique, l'étude des moteurs triphasés n'est pas obligatoire mais dépend du profil professionnel de la classe et se fait via une approche énergétique des machines, plutôt que fonctionnelle. La démarche d'investigation devient une référence centrale pour l'organisation des travaux pratiques : «le but des séances de travaux pratiques n'est pas exclusivement de vérifier, a posteriori, des lois ou des modèles théoriques. Il est aussi, d'apporter des connaissances en les faisant découvrir aux apprenants par une véritable démarche d'investigation. Il est souhaitable que ces pratiques soient mises en œuvre aussi souvent que possible». Les modalités de pédagogie par le

numérique sont également encouragés: «l'utilisation de l'outil informatique est recommandée ».

\subsection{Les sciences physiques et chimiques dans l'enseignement agricole : une discipline au service des disciplines techniques}

Depuis la création du premier référentiel modulaire avec le BTA en 1984, nous pouvons noter que l'enseignement des sciences physiques et chimiques est en tension entre un enseignement général et un enseignement technique. Cette discipline se retrouve mobilisée à la fois dans des 
modules communs à toutes les filières et dans des modules professionnels spécifiques. Elle est toutefois perçue dans les entretiens davantage comme un enseignement aux services des disciplines techniques qu'un enseignement général $\left(\mathrm{I}_{1}, \mathrm{I}_{3}\right.$ et $\left.\mathrm{I}_{6}\right)$.

Les sciences physiques et chimiques se retrouvent à l'interface de nombreuses disciplines scolaires et sont ainsi souvent dans des modules pluridisciplinaires, soit avec la biologieécologie, biochimie, microbiologie, en relation avec la chimie, soit avec l'agroéquipement pour la physique. Elles sont parfois associées aux mathématiques dans des modules scientifiques (MG4). On note également qu'elle peut prendre en charge toute seule un module d'enseignement technologique (par exemple M8 du STAV de 2013) ou professionnel (MP3 du bac professionnel de 1996).

L'analyse des grilles horaires montre qu'il y a eu une hausse des horaires des sciences physiques et chimiques jusqu'au milieu des années 90, puis à partir des années 2000, une baisse continue des heures d'enseignement à chaque rénovation.

Cette diminution peut paraître peu significative dans nos tableaux, mais ceux-ci sont réalisés à partir des horaires globaux du diplôme et ne tiennent pas compte de la répartition des filières dans les établissements : les établissements en fonction des profils de leurs filières ont pu être «perdants » (par exemple pour le passage du STPA au STAV) et d'autres " gagnants » (par exemple pour le passage des BTA production et transformation en Bac professionnel LCQ ou AEQ). Il faudrait étudier plus précisément la répartition des filières pour mieux se rendre compte de l'effet global des rénovations sur les horaires dans les établissements. Par contre, le passage du bac professionnel en 3 ans avec la disparition des BEPA, qui n'apparaît pas dans nos tableaux, a fait perdre une année de formation à tous les établissements, ce qui justifie les déclarations des inspecteurs : « la physique-chimie a baissé réforme après réforme » $\left(\mathrm{I}_{4}\right.$ et $\left.\mathrm{I}_{5}\right)$. L'écriture des programmes a de plus évolué : «les programmes on les fait et on les défait » $\left(\mathrm{I}_{6}\right)$. En effet, les prescriptions étaient très courtes dans les années $70\left(\mathrm{I}_{1}\right)$, puis elles ont été de plus en plus écrites en termes d'objectifs, progressions, commentaires, suggestions (« un outil confortable pour les enseignants mais aussi contraignants, avec peu de liberté pédagogique » selon $I_{1}$ ). L'écriture des programmes des rénovations actuelles retrouve à un nouveau une forme peu développée : «le référentiel actuel est limité en nombre de mots, 3 objectifs et 3 sous objectifs et le document d'accompagnement n'est pas obligatoire » $\left(\mathrm{I}_{6}\right)$. Une certaine liberté pédagogique est ainsi possible à travers la nécessité faite à l'enseignant d'adapter son enseignement au contexte : "il convient à chaque enseignant dans le respect du référentiel, d'adapter les exigences des contenus à traiter en fonction de la spécialité de la section dans laquelle il enseigne » (recommandations pédagogiques du MG4). En effet, il est bien stipulé que la spécialité professionnelle de la section induit l'approfondissement de certaines parties et ne nécessite qu'un développement succinct pour d'autres points de programme. Cette trajectoire actuelle des prescriptions tend donc à renforcer les sciences physiques et chimiques en tant que « discipline de service» (Martinand, 1992) des disciplines techniques.

Nous portons maintenant la discussion sur l'évolution de l'épistémologie scolaire de la discipline des sciences physiques et chimiques au travers des différents référentiels que nous venons de décrire.

\section{L'évolution de l'épistémologie scolaire de la discipline «sciences physiques et chimiques » de l'enseignement agricole}

Nous structurons cette partie en reprenant différentes dimensions de l'épistémologie scolaire selon Develay (1993).

\subsection{Evolution des connaissances déclaratives}


L'analyse des référentiels montre que certains savoirs à enseigner sont récurrents à travers les réformes et les filières, et constituent ainsi le fondement des connaissances disciplinaires : il s'agit de l'étude des solutions aqueuses en chimie (l'eau, l'acido-basicité, l'oxydoréduction) et de l'approche énergétique des phénomènes physiques.

D'autres savoirs disparaissent progressivement de la discipline scolaire des sciences physiques et chimiques pour être abordés dans d'autres disciplines scolaires. C'est par exemple le cas de la chimie du sol qui est maintenant faite par les agronomes ou des moteurs électriques qui sont étudiés en sciences et techniques des équipements. Ces changements montrent que les frontières entre les disciplines scolaires évoluent aussi : «les liens avec la biologie se sont distendus par rapport à ce qu'il y avait au départ. La chimie du sol a été prise par d'autres personnes, les agronomes et biologistes. Ce sont des glissements très doux » $\left(\mathrm{I}_{4}\right)$. Ces évolutions entre les disciplines conduisent également à reconfigurer l'approche des savoirs au sein d'une même discipline. C'est ainsi que l'étude de l'énergie chimique donnait du sens à une approche énergétique du vivant, alors qu'avec l'éloignement de la biologie, l'énergie chimique n'est maintenant plus un objet d'enseignement pour la physique. De même, les biomolécules sont associées dans les années 1980 au vivant (donc à la biologieécologie) alors qu'actuellement elles s'inscrivent dans des problématiques liées à l'alimentation (sans lien avec d'autres disciplines).

L'irruption de questionnements dans le champ social peut expliquer l'évolution des problématiques scolaires : la crise de la vache folle en 1996 et la question de la traçabilité des aliments ont pu influencer ce changement de regards sur les biomolécules. De même, l'appréhension de l'énergie par les enjeux énergétiques dans une perspective de développement durable rend compte d'une préoccupation sociale actuelle qui se propage dans le champ scolaire.

Cette présence de l'actualité est particulièrement visible pour le traitement scolaire du nucléaire: après avoir disparu des référentiels, il revient en 2013 dans le nouveau baccalauréat STAV, « il y a des effets de mode, par exemple quand on a remis le nucléaire on pensait qu'il y avait des choix de société qui se faisaient et les élèves devaient être au courant de quoi on parle sans se fier uniquement à ce qu'ils voient dans la presse. Le nucléaire avait complètement disparu » $\left(\mathrm{I}_{5}\right)$, «l'énergie nucléaire qui est un problème avec Tchernobyl, Fukushima etc. donc ça revient à la mode mais c'est normal c'est de l'éducation du citoyen » $\left(\mathrm{I}_{2}\right)$.

La prise en compte de l'actualité n'est pas la seule influence des changements dans les contenus, il y a aussi les arbitrages à faire entre les concepteurs des programmes, qui relèvent parfois de rapports de force : " en fonction de celui qui crie le plus fort quand on écrit le référentiel. Ce n'est pas seulement une histoire de personnes mais quand même. En classe prépa, c'était en fonction du dada des chercheurs qui écrivaient les programmes » $\left(\mathrm{I}_{5}\right)$.

Des savoirs disparaissent complètement et ne sont plus enseignés, tels que l'électromagnétisme, l'électronique et des éléments de physique moderne (les lois d'Einstein, la dualité ondes-corpuscules par exemple). Cette évolution suit également des effets de «mode »d'une époque : «dans les années 75 on ne jurait en physique que par les semiconducteurs, l'effet photoélectrique, les transistors, bref c'était la mode, ça n'a pas duré longtemps » $\left(\mathrm{I}_{2}\right)$.

De nouveaux savoirs apparaissent également, comme la prise en compte de la sécurité en physique (électricité), mais surtout en chimie : « une évolution qu'il faudra noter c'est celle de la sécurité et elle évolue toujours. On évolue toujours et c'est mieux comme ça. On distillait le mercure à l'époque » $\left(\mathrm{I}_{2}\right)$. En effet, la mise en place du règlement européen CLP (classification, labelling and packaging) et une note de service (DGER/SDPOFE/N20092089) qui en découle, permet aux établissements de l'enseignement agricole de prendre en 
compte le risque chimique par rapport aux nouvelles règles d'étiquetage des produits chimiques utilisés.

\subsection{Evolution des objets manipulés}

L'analyse des entretiens montre que l'arrivée d'un changement important dans l'enseignement a été l'apparition de nouveaux matériels pédagogiques $\left(\mathrm{I}_{1}, \mathrm{I}_{2}\right.$ et $\left.\mathrm{I}_{3}\right)$ comme la table à coussin d'air, qui a permis de renouveler l'approche expérimentale : «moi j'ai connu l'arrivée de la table à coussin d'air ça a été une révolution fantastique ça » $\left(\mathrm{I}_{5}\right)$.

L'EXAO (expérience assistée par ordinateur) est également un marqueur de l'évolution des outils manipulés par l'enseignant et les élèves. Selon $\mathrm{I}_{5}$, « on passait moins de temps à faire des mesures rébarbatives et qui prenaient beaucoup de temps, ce qui permettait de passer plus de temps sur des choses intéressantes » et le gain de temps permettait de travailler davantage le sens des activités faites : «la visualisation que tu en avais à l'ordinateur permettait de redonner du sens à ce qu'on enseignait ». De plus, outre le gain de temps obtenu, l'EXAO a permis de travailler des notions qui jusque là n'étaient pas perceptibles : « autre exemple qui m'a semblé très intéressant avec la venue de l'informatique : dans tout ce qui était force de frottement, il ne fallait surtout pas en parler, maintenant on arrive à faire calculer la force de frottement dans les liquides visqueux, ce sont des évolutions qui ont été très intéressantes » $\left(\mathrm{I}_{5}\right)$. Cet outil a marqué le début de l'ère numérique dans les classes, « l'EXAO a constitué un message fort de l'institution pour le développement du numérique » $\left(\mathrm{I}_{4}\right)$.

De même, à l'heure actuelle, le tableau blanc interactif commence à être utilisé dans l'enseignement des sciences physiques et chimiques, non sans interrogation sur les conséquences en termes de pédagogie $\left(\mathrm{I}_{5}\right)$.

Dans les témoignages, nous percevons qu'à travers l'évolution des objets manipulés, ce sont également les méthodes d'enseignement qui ont changé : si l'enseignement des sciences physiques et chimiques restent souvent magistral dans les pratiques observées $\left(\mathrm{I}_{6}\right)$, celles-ci ont tout de même évoluées pour rendre actifs les élèves, notamment par le développement des travaux pratiques.

\subsection{Evolution des tâches prescrites en lien avec les connaissances procédurales}

L'évolution des référentiels montre en effet un souci constant d'inscrire l'approche expérimentale au centre de l'enseignement des sciences physiques et chimiques, accordant ainsi une place plus importante au fait de rendre les élèves actifs.

Les témoignages des inspecteurs relient l'augmentation des travaux pratiques avec l'émergence de nouveaux matériels de laboratoire, mais aussi avec l'arrivée des CCF pratiques dans les diplômes de l'enseignement agricole $\left(\mathrm{I}_{3}\right.$ et $\left.\mathrm{I}_{5}\right)$ : « il y a eu un point qui était très intéressant : je me souviens quand on a commencé on faisait très peu de $\mathrm{TP}$ et petit à petit grâce aux CCF d'ailleurs la place des TP a été de plus en plus importante. Ca a été un grand changement dans l'enseignement de la physique-chimie » $\left(\mathrm{I}_{5}\right)$.

Le fait de réaliser des CCF pratiques a ainsi mis l'accent sur l'enseignement de compétences expérimentales qui sont pour Develay (1993) des connaissances procédurales (par exemple savoir faire un circuit électrique à partir d'un schéma, savoir faire un dosage), Ces connaissances nécessitent en amont des connaissances déclaratives, qui permettent d'effectuer la tâche (par exemple les conventions de représentation en électricité, des bases sur les solutions aqueuses). Progressivement, l'accent mis sur l'expérimental n'était plus confiné aux savoir-faire pratiques et à la reproduction de gestes techniques, une part de réflexion était demandée dans le cadre d'une pédagogie active et inductive $\left(\mathrm{I}_{1}\right)$. 
On observe que les référentiels précisent progressivement dans le temps les contours de la place de l'expérience. Ainsi, ils citent tout d'abord la place centrale accordée à la démarche expérimentale, sans plus de précisions (années 80), puis des capacités liées à l'élaboration d'hypothèses, au développement de l'esprit critique intègrent l'expérimentation (années 2000). Dans les dernières rénovations, les référentiels explicitent encore davantage la place de l'expérience et l'intègrent à la notion de méthode scientifique, dont l'aspect hypothéticodéductif est mis en avant en tant que démarche de construction et résolution de problèmes. La démarche d'investigation devient alors une méthode d'enseignement à privilégier. On perçoit donc que le rôle de l'expérience a évolué, s'est complexifié et s'est intégré à une démarche plus vaste qui relève de la méthode scientifique. Les connaissances procédurales ne sont donc plus uniquement expérimentales, mais à articuler avec l'expérience : les élèves doivent interpréter une expérience ou un protocole, argumenter, communiquer leur travail. Les connaissances déclaratives spécifiques à la discipline ne sont pas toujours indispensables pour fonder les connaissances procédurales, celles-ci sont maintenant référées à d'autres connaissances procédurales qui sont transversales (les compétences générales comme « communiquer ») ou bien liées à l'épistémologie de la discipline (le statut des hypothèses ou de l'expérience, le type de problématisation).

\subsection{Evolution de la matrice disciplinaire}

Notre étude nous indique des indices d'évolution de la matrice disciplinaire des sciences physiques et chimiques dans l'enseignement agricole.

Tout d'abord, la dimension expérimentale de cet enseignement s'est renforcée depuis les années 70 et elle s'est progressivement insérée comme élément, central mais non exclusif, des méthodes scientifiques. C'est ainsi que les connaissances procédurales se sont petit à petit affirmées, au détriment des connaissances déclaratives : on est passé d'un enseignement qui concevait son héritage culturel avant tout par la somme des connaissances produites (l'idée que la science consiste en des faits, des concepts, etc.) à la nécessité d'initier aux conditions de production de ces connaissances (l'idée que la science est une activité).

Cette évolution de type épistémologique s'inscrit bien entendu dans un climat favorable à cette éclosion : l'ouverture du champ scolaire à l'approche par compétences montre bien que les connaissances procédurales sont aujourd'hui privilégiées, car les connaissances déclaratives ne sont vues qu'à travers le prisme de leur mobilisation en situation.

Nous avons vu par ailleurs que les savoirs à enseigner dans la discipline des sciences physiques et chimiques se sont éloignés des disciplines biologie-écologie et agronomie (donc du vivant) et se sont rapprochées des disciplines liées aux techniques agroalimentaires ou aux équipements agricoles. A un moment où l'agro-écologie tend à devenir le nouveau paradigme du monde agricole, il y a un risque que les sciences physiques et chimiques soient marginalisées. Certains inspecteurs soulignent ainsi que « la chimie est devenue un gros mot » $\left(\mathrm{I}_{5}\right)$, « la physique-chimie est moins importante aux yeux de la société » $\left(\mathrm{I}_{4}\right)$.

L'évolution des liens avec les autres disciplines montre ainsi que les sciences physiques et chimiques sont une discipline «de service »: «nous, on consommait des maths mais nous on était consommé par tout le reste, l'agroalimentaire, l'agronomie, l'agroéquipement et surtout surtout la biologie » $\left(\mathrm{I}_{2}\right)$.

Si les changements de type épistémologiques interrogent la discipline, ce sont également les liens avec les autres disciplines qui sont remis en question. Le défi posé à la discipline des sciences physiques et chimiques consiste peut-être en sa capacité à investir la spécificité des connaissances procédurales dans les champs techniques, en proposant modèles et méthodes d'analyse à mobiliser en situation professionnelle. 


\section{Conclusion}

Nous avons poursuivi différents objectifs dans cette étude.

Notre démarche consiste tout d'abord en un effort de mémoire, afin de mieux comprendre les enjeux du présent, enjeux qui sont portés par une institution, dont le développement historique lui échappe en grande partie. En ce sens, cet objectif est atteint, le récit que nous avons construit est cohérent et conforme à la fois à la mémoire d'une partie de ceux qui ont vécu cette histoire et aux traces écrites (bien que partielles) que cette histoire nous a laissées. Bien sûr, nous avons écarté des dimensions importantes qui mériteraient une analyse plus profonde, comme par exemple une exploration de la mémoire des enseignants, de l'évolution de l'évaluation et de la certification, ou bien des archives de l'APEPA. De même, nous sommes parties prenantes de cette histoire et du processus historique continu, aussi l'angle que nous avons choisi traduit à la fois l'actualité des questions que nous nous posons (qui sont sans doute différentes de celles qui se poseront dans le futur) et notre évident manque de recul.

Nous avons cherché à suivre l'évolution de l'enseignement prescrit des sciences physiques et chimiques et il ressort de notre étude que cette discipline est «vivante », que ses liens avec le monde technique se reconfigurent, que les objets d'enseignement qui lui sont propres changent, que son indépendance vis-à-vis de l'éducation nationale est relative et changeante. L'explicitation de plus en plus prégnante de la méthode scientifique dans les prescriptions pose de plus la question de l'avenir de la discipline : il y a fort à parier que l'étape suivante soit des prescriptions qui intègrent des éléments d'épistémologie (c'est déjà en partie le cas avec la notion de modélisation), car si les capacités ou compétences propres à la démarche scientifique deviennent les objectifs d'enseignement/apprentissage, cela sous entend l'explicitation de la nature de cette démarche (par exemple la nature d'une hypothèse scientifique, la spécificité d'un problème scientifique, etc.). Ainsi, comme le rappelle Develay (1992, p. 17), «toute éducation de type scolaire suppose (...), au sein de la culture d'une époque donnée, une sélection de contenus destinés à être transmis aux générations nouvelles et qui constituent la culture scolaire (...) Les savoirs à enseigner constituent l'héritage qu'une génération souhaite léguer aux suivantes, le capital que les pères souhaitent transmettre à leurs enfants ». Cette «transmission d'héritage » pose également la question de la formation des enseignants, car comme le rappelle l'inspecteur $\mathrm{I}_{4}$, "enseigner c'est tellement complexe », qu'intégrer cette évolution de la discipline nécessite un apprentissage, dont nous espérons que notre étude historique constitue les prémisses d'un accompagnement. 


\section{Bibliographie}

APEPA (1973). Conditions de l'enseignement des sciences physiques dans l'enseignement technique agricole. Bulletin d'Union des Physiciens. 559, p. 259.

Assemblée Nationale, $\mathrm{n}^{\circ} 1548$ (2013). Projet de loi d'avenir pour l'agriculture, l'alimentation et la forêt, enregistré à la Présidence de l'Assemblée nationale le 13 novembre 2013

Consulté le 20 Janvier 2014, disponible à http://www.assembleenationale.fr/14/pdf/projets/pl1548.pdf

Bonneviale, J.-R. et Sebillote, M. (1985). Evolution de l'enseignement technique agricole de l'agriculture à l'agronomie. Bulletin de l'INRAP, 62, 35-45.

BUP (1977). La commission Lagarrigue. Supplément au Bulletin de l'Union des Physiciens. 597.

Chevallard, Y. (1991). La transposition didactique : du savoir savant au savoir enseigné. Grenoble : La Pensée Sauvage (1re éd. 1985).

Conseil européen de Lisbonne (2000). Conclusions de la Présidence, 23 et 24 mars 2000.

$\begin{array}{llllll}\text { Consulté } & \text { le } & 20 & \text { Janvier } & \text { 2014, à }\end{array}$ http://www.consilium.europa.eu/ueDocs/cms_Data/docs/pressData/fr/ec/00100-r1.f0.htm

D'Enfert, R. (2011). Projet REDISCOL. Réformer les disciplines scolaires : acteurs, contenus, enjeux, dynamiques (années 1950-années 1980). ANR-06-APPR-013.

Darré, J.P. (1985). L'automne des pédagogues peut-il contribuer à la prise en compte des diversités de l'agriculture ? in Ministère de l'Agriculture DGER ; Enseignements agricoles et formation des ruraux. Agri Nathan International, p. 156-157.

Dascon, F. (1985). L'école est-elle le seul lieu d'apprentissage du métier d'agriculteur ? in Ministère de l'Agriculture DGER; Enseignements agricoles et formation des ruraux. Agri Nathan International, p. 26-28.

Develay M. (1992). De l'apprentissage à l'enseignement. Paris : ESF.

Develay, M. (1993). Pour une épistémologie des savoirs scolaires. Pédagogie collégiale, 7-1, 35-40.

Direction Générale de l'Enseignement et de la Recherche (2010). Rapport de l'inspection de l'enseignement agricole 2009-2010. Dijon : Educagri éditions.

Direction Générale de l'Enseignement et de la Recherche (2013). Fiche de l'atelier du thème 2 «enseigner à produire autrement» du séminaire de lancement de la concertation pour l'avenir de l'Enseignement Agricole, vendredi 29 Mars 2013, consulté le 20 janvier 2014, disponible http://www.chlorofil.fr/systeme-educatif-agricole/organisation-orientations-etevolution-de-lea/concertation-pour-lavenir-de-lea/2-enseigner-a-produire-autrement.html 
Durkheim, E. (1922). Éducation et sociologie. Edition électronique, disponible http://classiques.uqac.ca/classiques/Durkheim_emile/education_socio/education_socio.html Consulté le 3 Février 2014.

Fourez, G. (1994). Alphabétisation scientifique et technique, essai sur les finalités de l'enseignement des sciences. Bruxelles : De Boeck Université.

Goudet, P. (1974). A propos de l'enseignement des sciences physiques. Bulletin de l' INRAP, Vol 18, p.19-20.

Goudet, P. \& Coudray, R. (1976) la méthode expérimentale et les méthodes pédagogiques en vigueur dans l'enseignement des sciences physiques. Bulletin de l' INRAP, Vol 50, p.21-61.

Goudet, P. (1981).Analyses des épreuves de sciences physiques. Bulletin de l' INRAP, Vol 18, p.19-20

Groupe Chapham (1980). A propos du contrôle des connaissances. Bulletin de l'Union des Physiciens. 627. 29-53.

Host, V. (1980). Note de synthèse La recherche pédagogique en sciences (Note limitée aux enseignements du 1er et du 2e degrés.). Revue française de pédagogie, 52, 79-90.

Hulin, N. (1991). La constitution et les débuts de la commission Lagarrigue (1969-1971) ou du rôle moteur des sociétés savantes. Bulletin d'Union des Physiciens. 730. 11-29.

Klatzmann J. (1953). La modernisation de l'agriculture. Revue économique. 5, 643-658.

Lelorrain A.-M. \& Bobbio, M. (2005). L'enseignement agricole et vétérinaire de la Libération à nos jours : textes officiels avec introduction, notes et annexes. Paris : INRP.

Martinand, J.-L. (1992). Enjeux et ressources de l'éducation scientifique. Introduction au thème. In A. Giordan, J.-L. Martinand et D. Raichvarg (Eds.) Actes des 14ème journées internationales sur la communication, l'éducation et la culture scientifiques et techniques. (pp. 57-65). Cachan : DIRES.

Méaille, M. \& Marshall, E. (1994). La formation professionnelle agricole. Tendances et perspectives. Didaskalia, 4, 87-97.

OCDE (1968). L'enseignement actuel de la physique.

Ricoeur, P. (1983). Temps et récit, tome 3. Paris : le seuil.

Rioux, J.-P. (1992). Peut-on faire une histoire du temps présent ? Questions à l'histoire des temps présents. Bruxelles : Complexe, 1992.

Robin, M. (1968). Au sujet des recherches américaines pour l'enseignement des Sciences Physiques, et de cet enseignement en France. Bulletin d'Union des Physiciens. 510, 541-548.

Roure, C. (2013). Épistémologie des savoirs scolaires et sens culturel des activités physiques et sportives, Carrefours de l'éducation, 35, 163-178. 
Secrétan, D. (1998). L'enseignement des sciences physiques dans les sections du baccalauréat professionnel : compte-rendu d'innovation. Didaskalia, 13, 145- 163.

Tardy, J. (1977). Vingt années d'enseignement technique agricole. Bulletin de l'INRAP, 32, 110.

Unesco (1966). Rapport Amélioration de l'enseignement des sciences, 28 février 1968. http://unesdoc.unesco.org/images/0014/001417/141789fb.pdf

Wolikow, S. (1998). L'histoire du temps présent en question. Bulletin de l'institut d'histoire contemporaine, 5(HS), 9-24. 